\title{
Some remarks on assigning weights to experts in multi-attribute group decision making using intuitionistic fuzzy sets
}

\author{
Eulalia Szmidt $^{1,2}$ and Janusz Kacprzyk $\mathbf{K}^{1,2}$ \\ ${ }^{1}$ Systems Research Institute, Polish Academy of Sciences \\ ul. Newelska 6, 01-447 Warsaw, Poland, and \\ ${ }^{2}$ Warsaw School of Information Technology \\ ul. Newelska 6, 01-447 Warsaw, Poland \\ e-mails: \{s zmidt, kacprzyk\} @ibspan.waw.pl
}

Received: 13 June 2020

Accepted: 20 September 2020

\begin{abstract}
We discuss how to assign weights to the experts participating in group decision making in intuitionistic fuzzy environment which means that the options are expressed via intuitionistic fuzzy sets (IFSs, for short). We use the three term representation of the IFSs. A question arises if by making use of the expert's opinions concerning a problem considered is it possible to assess the experts. The typical approaches from literature are recalled and discussed. Next, we propose two novel methods of assigning weights to experts. However, the methods are not ideal as starting from expert's opinions concerning the options considered. Alas, while not knowing a real solution of a problem the experts try to solve, it is difficult to tell who is right and who is wrong whereas we do not have additional knowledge about the experts. The advantage of the method proposed is that we avoid assumptions about a real optimal solution which is not known. Instead, we pay attention if an expert is able to tell in a convincing way which option is good and which one is bad by pointing out pros and cons of an option in a definite way.
\end{abstract}

Keywords: Intuitionistic fuzzy sets, Three term representation of IFSs, Multi-attribute group decision-making, Assigning weights to the experts.

2010 Mathematics Subject Classification: 03E72, 34Gxx. 


\section{Introduction}

Group decision making problems (GDM) are helpful in many aspects of our life starting from politics, science, technology, economy, education, and other fields. Each expert (decision maker) has his/her preferences to each option and the attribute considered. The opinions vary from expert to expert so they are aggregated and sometimes weights to the experts are assigned. In this paper we concentrate on assessing the experts. The weights of experts should be computed in an objective way. In the literature there are not too many ideas how to tackle the problem. Sometimes it is just assumed that the weights are predefined. An interesting approach is presented by Ramanathan and Ganesh [9] who proposed that each member of a group rates himself with others in terms of their relative importance. It means that the experts use their own subjective opinions. The idea boils down to an eigenvector based method working in one of the well known but specific techniques for decision making, namely Analytic Hierarchy Process AHP (cf. Saaty [11]).

Yang et al. [26] proposed to determine the weights of decision makers based on rough set theory (cf. Pawlak, Słowinski [8]). Yang et al. [26] derived a positive ideal solution (PIS) founded on the average matrix of rough group decision, and negative ideal solutions(NIS) founded on the lower and upper limit matrices of rough group decision. The weight of each group member was found out by using relative closeness method depending on the distances from each individual group member's decision to the PIS and NIS. The presented method [26] is useful for real number form of attributes only.

There are several other methods making use of the idea of PIS and NIS. For example Yue [27] proposed an extended TOPSIS method (Technique for Order Preference by Similarity to an Ideal Solution [7]) for ranking the order of decision makers. The distances from each individual decision to ideal decisions is the basis of assessing the experts. Next, Yue [28] assumed that the average decision of all individual decisions is the ideal decision. After that, the weight of expert is determined by the projection of individual decision on the ideal decision.

The cited above approaches make use of the expert's opinions concerning the options to assess the experts. In this paper we also make use of expert's opinions but we do not assume to know the ideal or real solution. We concentrate on the options from the point of view if the options expressed by using IFSs are properly described by experts in the sense of giving with confidence option's advantages and disadvantages.

\section{A brief introduction to IFSs}

One of the possible generalizations of a fuzzy set in $X$ (Zadeh [29]) given by

$$
A^{\prime}=\left\{\left\langle x, \mu_{A^{\prime}}(x)\right\rangle \mid x \in X\right\}
$$

where $\mu_{A^{\prime}}(x) \in[0,1]$ is the membership function of the fuzzy set $A^{\prime}$, is an IFS (Atanassov [1-3]) $A$ is given by 


$$
A=\left\{\left\langle x, \mu_{A}(x), \nu_{A}(x)\right\rangle \mid x \in X\right\}
$$

where: $\mu_{A}: X \rightarrow[0,1]$ and $\nu_{A}: X \rightarrow[0,1]$ such that

$$
0 \leq \mu_{A}(x)+\nu_{A}(x) \leq 1
$$

and $\mu_{A}(x), \nu_{A}(x) \in[0,1]$ denote a degree of membership and a degree of non-membership of $x \in A$, respectively (see Szmidt and Baldwin [13] for deriving memberships and non-memberships for A-IFSs from data).

An additional concept for each A-IFS in $X$, that is not only an obvious result of (2) and (3) but which is also relevant for applications, we will call (Atanassov [2])

$$
\pi_{A}(x)=1-\mu_{A}(x)-\nu_{A}(x)
$$

a hesitation margin of $x \in A$ which expresses a lack of knowledge of whether $x$ belongs to $A$ or not (cf. Atanassov [2]). It is obvious that $0 \leq \pi_{A}(x) \leq 1$, for each $x \in X$.

The hesitation margin turns out to be important while considering the distances (Szmidt and Kacprzyk [14, 15, 17], entropy (Szmidt and Kacprzyk [16, 18]), similarity (Szmidt and Kacprzyk [19]) for the IFSs, etc. i.e., the measures that play a crucial role in virtually all information processing tasks (Szmidt [12]).

The hesitation margin turns out to be relevant for applications - in image processing (cf. Bustince et al. [6]), the classification of imbalanced and overlapping classes (cf. Szmidt and Kukier [23-25]), the classification applying intuitionistic fuzzy trees (cf. Bujnowski [5]), group decision making (e.g., [4,20]), genetic algorithms [10], negotiations, voting and other situations (cf. Szmidt and Kacprzyk papers).

\section{Problem of group decision making}

Assume that there is a set of alternatives (options) $X=\left\{X_{1}, X_{2}, \ldots, X_{m}\right\}$, from which the most preferred alternative is to be selected by a group of $K$ experts (decision makers) $P=\left\{P_{1}, P_{2}, \ldots, P_{k}\right\}$. Suppose that the decision maker $P_{k}(k=1,2, \ldots, K)$ constructs IFS $X_{i j}^{k}=\left\{\left\langle X_{i}, \mu_{i, j}^{k}, \nu_{i, j}^{k}, \pi_{i, j}^{k}\right\rangle\right\}$ where $\mu_{i, j}^{k}, \nu_{i, j}^{k}, \pi_{i, j}^{k}$ are the degree of membership (or satisfaction), the degree of non-membership (or non-satisfaction), and the degree of hesitation margin (lack of knowledge) of the alternative $X_{i} \in X$ with respect to the attribute $A_{j} \in A=\left\{A_{1}, A_{2}, \ldots, A_{n}\right\}$ to the concept "excellence" given by $P_{k}$, respectively, and $0 \leq \mu_{i, j}^{k} \leq 1,0 \leq \nu_{i, j}^{k} \leq 1,0 \leq \pi_{i, j}^{k} \leq 1$, $\mu_{i, j}^{k}+\nu_{i, j}^{k}+\pi_{i, j}^{k}=1$. The bigger $\pi_{i, j}^{k}$ the higher a hesitation margin of the decision maker $P_{k}$ as to the "excellence" of the alternative $X_{i}$ with respect to the attribute $A_{j}$. Thus, a group decision making problem expressed in IFS environment can be expressed concisely as follows: 


$$
\begin{array}{c|cccc}
P^{k}= & A_{1} & A_{2} & \ldots & A_{n} \\
\hline X_{1} & \left\langle\mu_{11}^{k}, \nu_{11}^{k}, \pi_{11}^{k}\right\rangle & \left\langle\mu_{12}^{k}, \nu_{12}^{k}, \pi_{12}^{k}\right\rangle & \ldots & \left\langle\mu_{1 n}^{k}, \nu_{1 n}^{k}, \pi_{1 n}^{k}\right\rangle \\
X_{2} & \left\langle\mu_{21}^{k}, \nu_{21}^{k}, \pi_{21}^{k}\right\rangle & \left\langle\mu_{22}^{k}, \nu_{22}^{k}, \pi_{22}^{k}\right\rangle & \ldots & \left\langle\mu_{2 n}^{k}, \nu_{2 n}^{k}, \pi_{2 n}^{k}\right\rangle \\
\vdots & \vdots & \vdots & \ddots & \vdots \\
X_{m} & \left\langle\mu_{m 1}^{k}, \nu_{m 1}^{k}, \pi_{m 1}^{k}\right\rangle & \left\langle\mu_{m 2}^{k}, \nu_{m 2}^{k}, \pi_{m 2}^{k}\right\rangle & \ldots & \left\langle\mu_{m n}^{k}, \nu_{m n}^{k}, \pi_{m n}^{k}\right\rangle
\end{array}
$$

where $k=1, \ldots, K$.

As it was mentioned earlier, the problem of assigning weights to the experts has been considered in literature under different conditions as it is impossible to offer one general solution for purposes of all the considered decision making problems. Here we consider group decision making using IFSs. We assume that the experts represent the same status, i.e., there is not their boss or a very famous expert among them. However, the experience and confidence of the experts considering a concrete problem may be different. Some experts have more information than others (which is expressed here in terms of membership values $\mu$ and non-membership values $\nu$ ), and their confidence (expressed here as $1-\pi$ ) is also different. For example, if an expert $k$ assesses option $x_{i}$ taking into account attribute $j$ as $\langle 1,0,0\rangle$ means that an option is excellent and the expert is absolutely sure abut it. If an expert $k$ assesses option $x_{i}$ taking into account an attribute $A_{j}$ as $\langle 0.5,0.4,0.1\rangle$, it means that the option satisfies in opinion of the expert expectations to degree 0.5 , does not satisfy expectations to degree 0.4 , and the lack of knowledge of the expert is 0.1 .

The simplest method of assigning weights to the experts is to take into account hesitation margins the experts assign to the options. If an expert assigns high hesitation margins to the options considered it means that he/she is not sure whether an option is good or bad. In result a weight of the opinions given by such an expert should not be hight.

Example 3.1. Assume that expert $P_{1}$ gives opinion concerning three options $X_{i},(i=1, \ldots, 3)$ described via two attributes $A_{j},(j=1,2)$ in the following way:

$$
P_{1}=\begin{array}{c|cc} 
& A_{1} & A_{2} \\
\hline X_{1} & \langle 1,0,0\rangle & \langle 0,1,0\rangle \\
X_{2} & \langle 0.7,0.2,0.1\rangle & \langle 0.9,0.1,0\rangle \\
X_{3} & \langle 0.5,0.4,0.1\rangle & \langle 0.2,0.8,0\rangle
\end{array}
$$

Option $X_{1}$ got two opposite opinions (very good, and very bad) in respect of the two attributes considered. But in both cases the hesitation margin is equal to 0, i.e., the expert is sure about the two opinion and the average score $S$ with respect to hesitation margin is 1 . In general we use

$$
S^{k}\left(X_{i}\right)=\frac{1}{n} \sum_{i=1}^{n}\left(1-\pi_{X_{i}}\right)
$$

$k=1, \ldots, K$. In our Example the scores in respect to the options are: $S^{1}\left(X_{1}\right)=\frac{1}{2}(1+1)=1$, $S^{1}\left(X_{2}\right)=\frac{1}{2}(0.9+1)=0.95, S^{1}\left(X_{3}\right)=\frac{1}{2}(0.9+1)=0.95$. 
In result expert $P_{1}$ gets:

$$
S^{1}(X)=\sum_{i=1}^{m} S^{1}\left(X_{i}\right)
$$

In Example 3.1 we have $S^{1}(X)=1+0.95+0.95=2.9$.

Assume that a second expert $P_{2}$ delivered the following opinions concerning the same options and attributes:

$$
P_{2}=\begin{array}{c|cc} 
& A_{1} & A_{2} \\
\hline X_{1} & \langle 0.8,0,0.2\rangle & \langle 0.3,0.5,0.2\rangle \\
X_{2} & \langle 0.7,0.1,0.2\rangle & \langle 0.6,0.3,0.1\rangle \\
X_{3} & \langle 0.6,0.4,0\rangle & \langle 0.1,0.7,0.2\rangle
\end{array}
$$

Repeating the same steps as previously we obtain from (5): $S^{2}\left(X_{1}\right)=\frac{1}{2}(0.8+0.8)=0.8$, $S^{2}\left(X_{2}\right)=\frac{1}{2}(0.8+0.9)=0.85, S^{2}\left(X_{3}\right)=\frac{1}{2}(1+0.8)=0.9$, and from $(6): S^{2}(X)=0.8+0.85+$ $0.9=2.55$.

To assign weights $w_{k},(k=1, \ldots, K)$ to experts where $0 \leq w_{k} \leq 1$, and $\sum_{k=1}^{K} w_{k}=1$ we normalize the scores $S^{k}(X), k=1, \ldots, K$, namely:

$$
w_{k}=\frac{S^{k}(X)}{\sum_{k=1}^{K} S^{k}(X)}
$$

For the data from Example 3.1 the weights are $w_{1}=2.9 /(2.9+2.55)=0.53$, $w_{2}=2.55 /(2.9+2.55)=0.47$ which means that Expert 1 has a little bit higher weight than Expert 2.

Following the literature trends of assigning the weights to experts by taking into account their preferences concerning options considered, we propose another, more detailed method. So far we were taking into account only the hesitation margins assigned to options given by the experts. Now we will take into account also membership values and non-membership values. We assume that the best opinions (reflecting how good are experts) should make it possible to conclude with confidence if an option is good or bad. Such condition is fulfilled when membership values are as far as possible from non-membership values, and as previously, the hesitation margin is as small as possible. The same conditions were formulated by Szmidt and Kacprzyk [21], Szmidt et al. [22] but used in a different context (attribute selection). The simplest function $f^{k}\left(X_{i}\right)$ fulfilling the above condition for an expert $k$ and a single option $X_{i}$ is:

$$
f^{k}\left(X_{i}\right)=\frac{1}{n} \sum_{j=1}^{n}\left(1-\pi_{i j}\right)\left(\left|\mu_{i j}-\nu_{i j}\right|\right)
$$

The properties of (8) are:

1. $0 \leq f^{k}\left(X_{i}\right) \leq 1$.

2. $f^{k}\left(X_{i}\right)=\left(f^{k}\left(X_{i}\right)^{C}\right)$ 
3. For a fixed value of $\left.\left|\overline{\mu_{i}^{k}}-\overline{\nu_{i}^{k}}\right|,\left(f^{k}\left(X_{i}\right)\right)\right)$ increases while $\pi_{i}^{k}$ decreases.

4. For a fixed value of $\pi_{i}^{k}, f^{k}\left(X_{i}\right)$ behaves dually to a very simple sort of entropy measure $\left|\overline{\mu_{i}^{k}}-\overline{\nu_{i}^{k}}\right|$ (i.e., as $1-\left(\left|\overline{\mu_{i}^{k}}-\overline{\nu_{i}^{k}}\right|\right)$ ).

In result of using (8) expert $P_{k}$ assessing all the options receives the score:

$$
f^{k}(X)=\sum_{i=1}^{m} f^{k}\left(X_{i}\right)
$$

Finally, to assign weights $w_{k},(k=1, \ldots, K)$ to experts where $0 \leq w_{k} \leq 1$, and $\sum_{k=1}^{K} w_{k}=1$ we normalize the scores $f^{k}(X), k=1, \ldots, K$, namely:

$$
w_{k}=\frac{f^{k}(X)}{\sum_{k=1}^{K} f^{k}(X)}
$$

Using the method (8) - (10) we obtain the following results for the data in Example 3.1.

From (8) we have:

$$
\begin{aligned}
& f^{1}\left(X_{1}\right)=\frac{1}{2}((1-0)|1-0|+(1-0)|0-1|)=1 \\
& f^{1}\left(X_{2}\right)=\frac{1}{2}((1-0.1)|0.7-0.2|+(1-0)|0.9-0.1|)=0.625 \\
& f^{1}\left(X_{3}\right)=\frac{1}{2}((1-0.1)|0.5-0.4|+(1-0)|0.2-0.8|)=0.345 \\
& f^{2}\left(X_{1}\right)=\frac{1}{2}((1-0,2)|0.8-0|+(1-0.2)|0.3-0.5|)=0.4 \\
& f^{2}\left(X_{2}\right)=\frac{1}{2}((1-0.2)|0.7-0.1|+(1-0.1)|0.6-0.3|)=0.375 \\
& f^{2}\left(X_{3}\right)=\frac{1}{2}((1-0)|0.6-0.4|+(1-0.2)|0.1-0.7|)=0.34 .
\end{aligned}
$$

From (9):

$$
\begin{aligned}
& f^{1}(X)=1+0.625+0.345=1.97 \\
& f^{2}(X)=0.4+0.375+0.34=1.115
\end{aligned}
$$

From (10):

$$
w_{1}=\frac{1.97}{1.97+1.115}=0.639, \quad w_{2}=\frac{1.115}{1.97+1.115}=0.361
$$

which means that from our approach (8)-(10) it turns out that expert $P_{1}$ is more important that expert $P_{2}$.

In this way we have proposed two methods of assigning the weights to experts in group decision making. Our starting point was similar as in some other methods known from literature - 
the experts are being assessed making use of their opinions they assign to the options considered as we do not have other data to use. However, a question arises if assessing experts this way is reliable. The problem is that we do not know the real state of affairs, i.e., we do not know a real the best option(s). The methods taking into account experts' opinions assume that an expert who gives with confidence very good scores or very bad scores is a very good expert. However, it could happen that the options considered are average and the best expert who knows about it gives average scores. On the other hand a less experienced expert might give very good scores and in result such an expert is seen as a very good one which is not the truth in the considered case. To sum up, we should be very careful assigning weights to experts when the only information about them are their opinions concerning the options.

\section{Conclusions}

We have proposed a novel method for assigning the weights to experts in a group decision process. We used three term model of IFSs which made it possible to formulate an understandable function being a foundation of the proposed algorithm. We avoided an assumption that we know an optimal solution (resulting e.g., from all expert's opinions producing an average opinion and using it as optimal one). Instead we assigned higher weights to the experts who are able to point out advantages and disadvantages of the options considered in a definite way. The method is transparent and simple from the point of view of calculations. It was not compared to other methods as in our opinion only real experiments could confirm it in a reliable way.

\section{References}

[1] Atanassov, K. (1983). Intuitionistic Fuzzy Sets. VII ITKR Session. Sofia (Centr. Sci.-Techn. Libr. of Bulg. Acad. of Sci., 1697/84) (in Bulgarian).

[2] Atanassov, K. (1999). Intuitionistic Fuzzy Sets: Theory and Applications. Springer-Verlag.

[3] Atanassov, K. (2012). On Intuitionistic Fuzzy Sets Theory. Springer-Verlag.

[4] Atanassova, V. (2004). Strategies for Decision Making in the Conditions of Intuitionistic Fuzziness. Int. Conf. 8th Fuzzy Days, Dortmund, Germany, 263-269.

[5] Bujnowski, P., Szmidt, E., Kacprzyk, J. (2014). Intuitionistic Fuzzy Decision Trees - a new Approach. In: Rutkowski L., Korytkowski M., Scherer R., Tadeusiewicz R., Zadeh L., Zurada J. (Eds.): Artificial Intelligence and Soft Computing, Part I. Springer, Switzerland, 181-192.

[6] Bustince, H., Mohedano, V., Barrenechea, E., Pagola, M. (2006). An algorithm for calculating the threshold of an image representing uncertainty through A-IFSs. IPMU'2006, 2383-2390. 
[7] Hwang, C. L., \& Yoon, K. (1981). Multiple Attribute Decision Making Methods and Applications, Springer-Verlag, Berlin.

[8] Pawlak, Z., \& Słowinski, R. (1994). Rough set approach to multi-attribute decision analysis. European Journal of Operational Research, 72, 443-459.

[9] Ramanathan, R., \& Ganesh, L. S. (1994). Group preference aggregation methods employed in AHP: An evaluation and an intrinsic process for deriving members' weightages. European Journal of Operational Research, 79, 249-265.

[10] Roeva, O., \& Michalikova, A. (2013). Generalized net model of intuitionistic fuzzy logic control of genetic algorithm parameters. Notes on Intuitionistic Fuzzy Sets, 19 (2), 71-76.

[11] Saaty, T. L. (1980). The Analytic Hierarchy Process, McGraw- Hill, New York.

[12] Szmidt, E. (2014). Distances and Similarities in Intuitionistic Fuzzy Sets. Springer.

[13] Szmidt, E., \& Baldwin, J. (2006). Intuitionistic Fuzzy Set Functions, Mass Assignment Theory, Possibility Theory and Histograms. 2006 IEEE World Congress on Computational Intelligence, 237-243.

[14] Szmidt, E., \& Kacprzyk, J. (1997). On measuring distances between intuitionistic fuzzy sets, Notes on Intuitionistic Fuzzy Sets, 3 (4), 1-13.

[15] Szmidt, E., \& Kacprzyk, J. (2000). Distances between intuitionistic fuzzy sets, Fuzzy Sets and Systems, 114 (3), 505-518.

[16] Szmidt, E., \& Kacprzyk, J. (2001). Entropy for intuitionistic fuzzy sets. Fuzzy Sets and Systems, 118 (3), 467-477.

[17] Szmidt, E., \& Kacprzyk, J. (2006). Distances Between Intuitionistic Fuzzy Sets: Straightforward Approaches May Not Work. IEEE Intelligent System'06, London, 716-721.

[18] Szmidt, E., \& Kacprzyk, J. (2007). Some problems with entropy measures for the Atanassov intuitionistic fuzzy sets. Applications of Fuzzy Sets Theory. LNAI 4578, 291-297.

[19] Szmidt, E., \& Kacprzyk, J. (2007). A New Similarity Measure for Intuitionistic Fuzzy Sets: Straightforward Approaches May Not Work. 2007 IEEE Conf. on Fuzzy Systems, 481-486.

[20] Szmidt, E., \& Kacprzyk, J. (2009). Ranking of Intuitionistic Fuzzy Alternatives in a Multi-criteria Decision Making Problem. In: Proceedings of the conference: NAFIPS 2009, Cincinnati, USA, June 14-17, 2009, IEEE, ISBN: 978-1-4244-4577-6.

[21] Szmidt, E., \& Kacprzyk, J. (2018). Selection of the attributes in intuitionistic fuzzy models. Notes on Intuitionistic Fuzzy Sets, 24 (4), 63-71, DOI: 10.7546/nifs.2018. 
[22] Szmidt E., Kacprzyk, J., \& Bujnowski, P. (2020) Attribute Selection for Sets of Data Expressed by Intuitionistic Fuzzy Sets. 2020 IEEE International Conference on Fuzzy Systems (FUZZ-IEEE), 1-7.

[23] Szmidt, E., \& Kukier, M. (2006). Classification of Imbalanced and Overlapping Classes using Intuitionistic Fuzzy Sets. IEEE IS'06, London, 722-727.

[24] Szmidt, E., \& Kukier, M. (2008). A New Approach to Classification of Imbalanced Classes via Atanassov's Intuitionistic Fuzzy Sets. In: Hsiao-Fan Wang (Ed.): Intelligent Data Analysis : Developing New Methodologies Through Pattern Discovery and Recovery. Idea Group, 85-101.

[25] Szmidt, E., \& Kukier, M. (2008) Atanassov's intuitionistic fuzzy sets in classification of imbalanced and overlapping classes. In: Panagiotis Chountas, Ilias Petrounias, Janusz Kacprzyk (Eds.): Intelligent Techniques and Tools for Novel System Architectures. Springer, Berlin Heidelberg, 455-471.

[26] Yang, Q., Du, P.-A., Wang, Y., \& Liang, B. (2017). A rough set approach for determining weights of decision makers in group decision making. PLoS ONE 12(2): e0172679. doi:10.1371/journal.pone.0172679.

[27] Yue, Z. L. (2013). Group decision making with multi-attribute interval data. Information Fusion, 14, 551-561.

[28] Yue, Z. L. (2012). Approach to group decision making based on determining the weights of experts by using projection method. Applied Mathematical Modelling, 36, 2896-2906.

[29] Zadeh, L. A. (1965). Fuzzy sets. Information and Control, 8, 338-353. 